\title{
BRONGNIARTIA MONTALVOANA (FABACEAE: FABOIDEAE), UNA ESPECIE NUEVA DE LA CUENCA DEL RIO BALSAS
}

\author{
Oscar Dorado y Dulce M. Arias \\ Rancho Santa Ana Botanic Garden \\ Claremont, California 91711-3101, U.S.A. \\ y \\ Universidad Autónoma del Estado de Morelos \\ Ave. Universidad \#1001, Cuernavaca, Morelos, México
}

\begin{abstract}
RESUMEN
Se describe e ilustra una especie nueva de la familia Fabaceae, Brongniartia montalvoana, de la Cuenca del Río Balsas en los estados de Morelos, Guerrero y Puebla. Se discuten sus afinidades con B. mortonii McVaugh, asi como las características morfológicas que las distinguen. Asimismo, se proporcionan datos de su hábitat.
\end{abstract}

\begin{abstract}
A new species of Fabaceae, Brongniartia montalvoana, from the Rio Balsas Basin is described and illustrated. Its affinities with $B$. mortonii McVaugh as well as their distinguishing morphological characteristics are discussed and habitat data are presented.

Brongniartia (Fabaceae: Faboideae) incluye alrededor de 60 especies de plantas leñosas distribuidas primordialmente en la parte noroeste y sur de México (Dorado, 1987). Estudios taxonómicos en el género han ocasionado que el número de especies se haya incrementado de manera considerable en los últimos 3 años. McVaugh (1987) recientemente describió 7 entidades nuevas en la flora de la región de Nueva Galicia. Dorado $(1987,1989)$ dió a conocer dos especies nuevas, una de la región del Istmo de Tehuantepec en Oaxaca (B. sousae) y la otra endémica del estado de Morelos (B. vazquezii). Jiménez y Contreras (1989) describieron una más del estado de Guerrero (B. guerrerensis). Como parte de un estudio sistemático del género Brongniartia, se han encontrado varias colecciones de la parte sur de México, las cuales no corresponden con ninguno de los taxa conocidos en la actualidad y en este trabajo se describen como una especie nueva.
\end{abstract}

Brongniartia montalvoana O. Dorado et D. M. Arias, sp. nov. (Fig. 1).

Brongniartia mortonii McVaugh affinis, sed habito arboreo ad $6.5 \mathrm{~m}$ alto, cortice squamato, foliolis (2.3) $3-7$ (9.3) cm longis, (1.1) 1.6-4 (5.7) cm latis et floribus marroninis pallidis differens. 
Arbol pequeño de hasta $6.5 \mathrm{~m}$ de alto; la corteza grisácea y comunmente escamosa; ramas maduras grisáceas, glabras o esparcidamente pilosas, ramas nuevas canescentes o tomentulosas. Estípulas $1.0-3.1 \mathrm{~cm}$ de largo, $(0.23) 0.5-1.1 \mathrm{~cm}$ de ancho, oblicuamente ovadas a muy ampliamente ovadas, u oblicuamente lanceoladas a linear lanceoladas, por lo general deciduas en hojas maduras, tomentulosas o esparcidamente pilosas, más densamente en la superficie abaxial y a lo largo de la vena principal; venación por lo común conspicua, con tricomas blanquecinos o de color pardo oscuro, textura más bien coriácea; lóbulo inferior formando un ángulo de $10^{\circ}$ a $160^{\circ}$ con el eje longitudinal de la estípula, con la base cordada o algunas veces aguda; lobulo superior ausente o bien desarrollado y entonces formando un ángulo de $-145^{\circ}$ a $-180^{\circ}$ con el eje longitudinal de la estípula, el margen de la estípula adyacente al pecíolo incurvado o excurvado, ápice agudo u obtuso. Hojas (2.1) $8-28 \mathrm{~cm}$ de largo; peciolo $1.7-2.5 \mathrm{~cm}$ de largo; raquis (1.8) 6.0-20 cm de largo, con 11-19 folíolos opuestos; estipelas $2.8-3.2 \mathrm{~mm}$ de largo, 0.6-0.7 $\mathrm{mm}$ de ancho en la base, rojo amarillentas, gruesas y duras; folíolos (2.3) 3.0-7.0 (9.3) $\mathrm{cm}$ de largo, (1.1) 1.6-4.0 (5.7) cm de ancho; lámina ovada, elíptica u oblonga, textura más bien coriácea, tomentulosa o esparcidamente pilosa, con tricomas blanquecinos o de color castaño oscuro, hasta de $0.5 \mathrm{~mm}$ de largo, por lo común más densamente distribuidos en la superficie abaxial, base redondeada o ligeramente cordada, ápice redondeado, obtuso o agudo, con un mucrón de 0.5-2.0 mm de largo; peciólulo 1.5-2.5 (4.5) mm de largo. Flores desarrollándose en ramas (hasta de $30 \mathrm{~cm}$ de largo) sin hojas (aparentando ser racimos), solitarias o $2-3$ por nudo; pedicelos $(0.5) \quad 0.8-2.8 \mathrm{~cm}$ de largo, erectos, tomentulosos o canescentes, con tricomas $0.6-1.3 \mathrm{~mm}$ de largo, blanquecinos o pardos. Bracteolas ausentes, en su lugar existe pubescencia más densamente distribuida (en la unión del cáliz con el pedicelo); cáliz 1.6-2.2 cm de largo, 6-9 mm de ancho, campanulado, tomentuloso, con tricomas de hasta $1 \mathrm{~mm}$ de largo y de color pardo oscuro; los dos lóbulos superiores (7.5) 10-13 $\mathrm{mm}$ de largo, (5.8) 6.3-7.0 mm de ancho, connados $1 / 3$ a $1 / 4$ de su longitud, lóbulos laterales (0.75) $0.9-1.3 \mathrm{~cm}$ de largo, (3.0) $4.5-6.0 \mathrm{~mm}$ de ancho, triangulares, lóbulo inferior (0.7) $1.0-1.3 \mathrm{~cm}$ de largo, (3.0) $3.5-5.0 \mathrm{~mm}$ de ancho, angostamente triangular; pétalos de color marrón pálido a rosado; estandarte $2.1-2.6 \mathrm{~cm}$ de largo (incluyendo la uña), 2.2-2.6 cm de ancho, reflejo formando un ángulo de casi $90^{\circ}$, mácula verde-amarillenta, lámina del estandarte ampliamente ovada u orbicular, algunas veces trapezoidal, emarginada en el ápice, auriculada en la base, uña 2.0-2.2 mm de largo, 2.0-2.2 mm de ancho, fuertemente curvada; alas (18) 20-22 mm de largo (incluyendo la uña), (8.0) 8.5-9.5 mm de ancho, aurícula formando con la uña un ángulo de $100^{\circ}$ a $125^{\circ}$, uña 3.0-3.2 mm de largo, 1.5-1.7 mm de ancho; quilla 19-21 mm de largo, (7.0) 8.0-9.5 mm de ancho, margen ventral de la quilla excurvado, recto o incurvado, uña 3-4 mm de largo, 1.2-1.4 mm de ancho, aurícula de la quilla formando con la uña un ángulo de $100^{\circ}$ a $120^{\circ}$; androceo $2.2-2.8 \mathrm{~cm}$ de largo, anteras 2.0-2.2 $\mathrm{mm}$ de largo; ovario 2.4$2.5 \mathrm{~cm}$ de largo, con tricomas hialinos principalmente a lo largo de las suturas, con un disco estaminal negruzco en la base del ovario de $0.4-0.5 \mathrm{~mm}$ de largo, estilo $1.0-1.2 \mathrm{~cm}$ de largo; ovvulos 7-8. Fruto (5.7) $6.4-8.3 \mathrm{~cm}$ de largo, $1.5-1.9 \mathrm{~cm}$ de ancho, oblongo 0 algunas veces oblanceolado, estípite $3-6 \mathrm{~mm}$ de largo, mucrón 1.6-4.0 mm de largo, ala del fruto $0.5-0.9 \mathrm{~mm}$ de ancho. Semillas $6-8$ por fruto, $8.2-8.7 \mathrm{~mm}$ de largo, $5.4-6.4 \mathrm{~mm}$ de ancho, elipsoides, pardas o pardas obscuras, con manchas negras. 
Dorado y Arias: Brongniartia montalvoana, una Especie Nueva de la Cuenca del Río Balsas

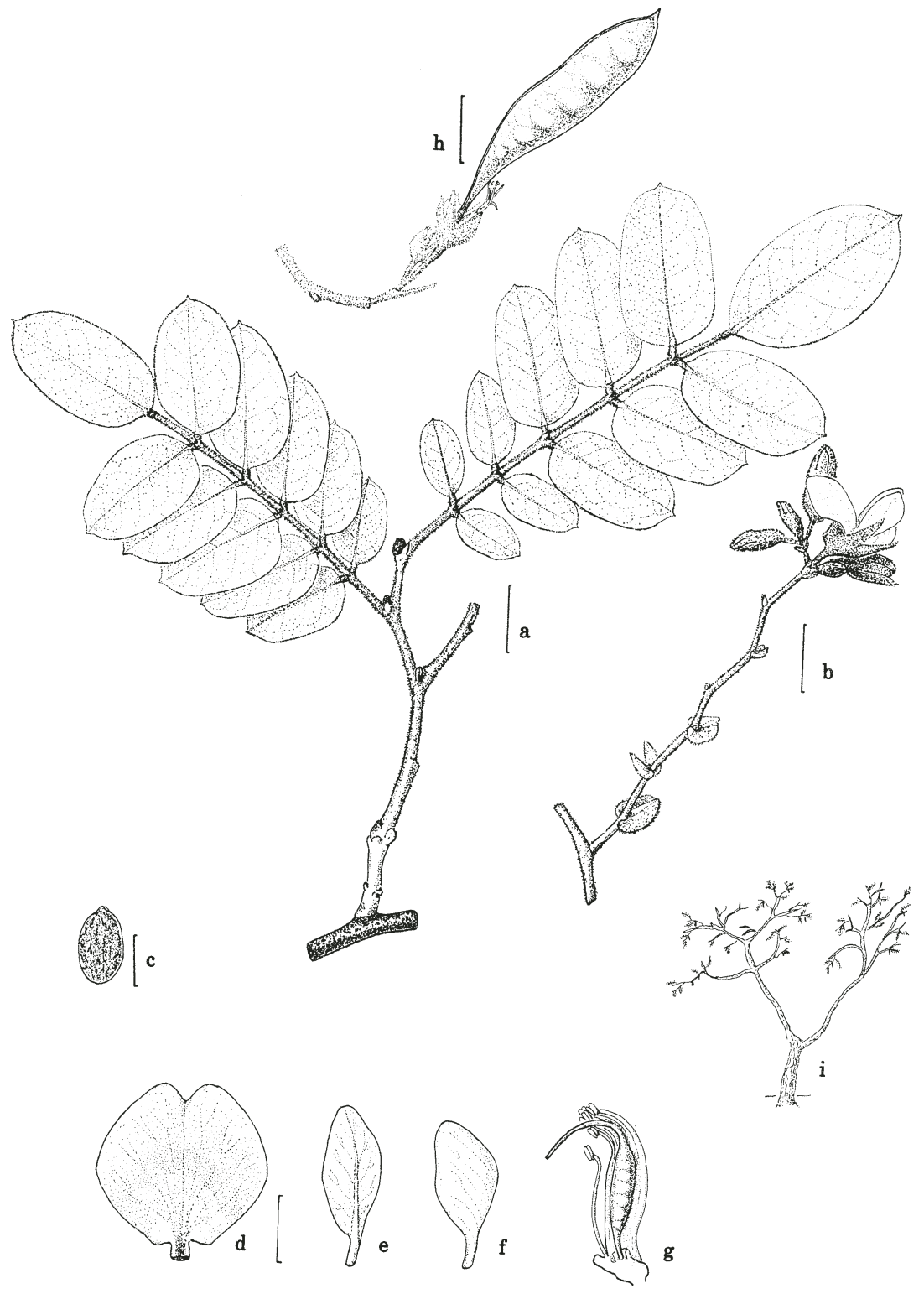

Fig. 1. Brongniartia montalvoana O. Dorado et D. M. Arias. a. Rama con hojas (basado en Dorado 1737 ); b. Rama con flores (basado en el holotipo); c. Semilla (basado en Elias 10646 ); d. Estandarte; e. Ala; f. Quilla (d-f basados en Sousa 3801); g. Gineceo y corte longitudinal del androceo (basado en Elias 10646); $h$. Fruto (basado en Anderson et al. 5680 ) Escala $=2 \mathrm{~cm}$ en $\mathrm{a}-\mathrm{b} ; 5 \mathrm{~cm}$ en c; $1 \mathrm{~cm}$ en d-g; $2 \mathrm{~cm}$ en h. i. Planta adulta. 
TIPO: México, Morelos, municipio Tlaquiltenango, Cerro Las Chinancas, $12 \mathrm{~km}$ al SE de Tlaquiltenango, 11.Il.1983. T. Wendt, M. Vázquez, T. Atkinson y M. Atkinson 4044 (MEXU).

Material adicional examinado: PUEBLA: municipio de Tehuitzingo, $12 \mathrm{~km}$ SE of Izúcar de Matamoros by the road to Tehuitzingo, 22.VII.1988, O. Dorado y D. Arias 1909a (RSA); Acatlán, 9.X.1942, F. Miranda 2447 (MEXU); Costa Palma, 22.XII.1942, F. Miranda 2560 (MEXU); Cerro Tlaac, 23.XII.1942, F. Miranda 2569 (MEXU); Izúcar de Matamoros, 26.III.1943, F. Miranda 2755 (MEXU); Cerro Tepunastli, 25.XII.1942, F. Miranda 2580 (MEXU); Cerro Teyuca, Atlixco-Izúcar de Matamoros, 4.Il.1973, W. Boege 2718 (MEXU). MORELOS: municipio de Tlaquiltenango, $12 \mathrm{~km} \mathrm{SE}$ of Tlaquiltenango on $E$ slope of Cerro Las Chinancas, 17.VII.1987, O. Dorado 1712, S. Zona, A. Burgos y R. Ramírez (RSA); ibid., 1.XII.1987, T. Elias 10644, 10645, 10646 (RSA). GUERRERO: $3 \mathrm{~km}$ SW of Xochipala, 10.Il.1970, W. Anderson 5680 y C. Anderson (MICH); municipio Chichihualco, 14 miles SW of Xochipala on the road toward Filo de Caballo, 13.VII.1987, O. Dorado et al. 1737 (RSA); $11 \mathrm{~km}$ SE of Huitzuco toward Atenango del Río, 12.VII.1987, O. Dorado et al. 1740 (RSA); Huiziltepec, antes de Zumpango del Río, 2.l.1971, E. Halbinger 404 (MEXU); Atetetla, 29.V.1976, A. Delgado 157, A. Campomanes y O. Téllez (MEXU); Tixtla, Chilapa, 16.I.1946, F. Miranda 3977 (MEXU); $10 \mathrm{~km}$ al SO de Xochipala, carretera a Filo de Caballo, 2.I.1983, J. Soto 5163, E. Martínez y T. Ramamoorthy (MEXU); between Chilpancingo and Mazatlán, 10.I.1977, W. Shwabe s.n. (MEXU); Totoapan, $20 \mathrm{~km}$ al noroeste de Iguala, 20.VII.1969, M. Sousa 3801 (MEXU); 8 km después de Xochipala, sobre la carretera Xochipala-Filo de Caballo, 31.V.1980. L. Valladares 12, H. Cruz y E. Castro (MEXU).

Brongniartia montalvoana es una especie muy vistosa debido al gran tamaño de sus flores y al color marrón claro a rosa de los pétalos, lo cual es poco común en el género. Brongniartia montalvoana presenta afinidades morfológicas con B. mortonii. Ambas pertenecen al grupo Podalyrioides (Dorado, 1988), caracterizado por la presencia de estípulas cordadas o reniformes normalmente grandes y bracteolas muy reducidas y filiformes. Las dos especies comparten varios rasgos morfológicos, entre los que se encuentra la pubescencia de los folíolos, la ausencia o reducción en el tamaño de las estipelas, la pubescencia en la base del cáliz, el tamaño de las flores, frutos y semillas, así como el número de semillas por fruto. Todas estas similitudes destacan la íntima relación que existe entre ambos taxa, que a su vez difieren entre si en las características que a continuación se ordenan en forma de clave dicotómica:

1 Flores de color azul claro, arbustos hasta de $2 \mathrm{~m}$ de alto, corteza lisa, folíolos (1.3) 2.0-5.4 cm de largo, (0.3) 0.8-3.2 cm de ancho; Jalisco y Michoacán ... B. mortonii 1 Flores de color marrón pálido a rosado, árboles pequeños hasta de $6.5 \mathrm{~m}$ de alto, corteza escamosa, folíolos (2.3) 3.0-7.0 (9.3) cm de ancho; Morelos, Puebla y Guerrero B. montalvoana

Brongniartia montalvoana se distribuye en la región central de la cuenca del Río Balsas, en la parte sur de Morelos, suroeste de Puebla y en la zona norte y central de Guerrero a altitudes de 900-1500 m. El hábitat de B. montalvoana es más diverso que 
el de B. mortonii, su especie más afín. Esta última normalmente se encuentra en zonas de transición entre bosque de encino y bosque tropical subcaducifolio en la región centrosur de Jalisco, mientras que $B$. montalvoana se desarrolla en bosques de encino, bosque tropical caducifolio y en palmares de Brahea en lugares con suelos calizos de la región del Río Balsas. Entro las especies más comunmente asociadas con la que aquí se describe se encuentran Pseudosmodingium perniciosum (Kunth) Engl., Lysiloma acapulcense (Kunth) Benth. y varios representantes de tallo exfoliante del género Bursera. El período de floración se presenta de diciembre a marzo. Brongniartia montalvoana con frecuencia llega a alcanzar alturas de $6.5 \mathrm{~m}$ y se le ha encontrado como uno de los árboles más abundantes, especialmente en lugares con bosque tropical caducifolio. El hábito arbóreo es relativamente poco común en el género, puesto que sólo se conoce otra especie de esta forma biológica (no relacionada con B. montalvoana), que se localiza en el estado de Oaxaca (Dorado y Torres, en preparación).

El nombre de esta elegante planta está dedicado al Dr. Alejandro M. Montalvo Pérez, rector de la Universidad de Morelos, quien durante su gestión administrativa ha apoyado notablemente las investigaciones biológicas en el estado de Morelos. El Dr. Montalvo se encuentra activamente trabajando en proyectos de conservación, en especial en la creación de una reserva ecológica en el sur del estado, en un área de bosque tropical caducifolio, ecosistema que está siendo severamente alterado en México y que es hábitat natural de esta especie.

\section{AGRADECIMIENTOS}

Agradecemos a H. Cota, A. Delgado, T.S. Elias, M. Sousa y O. Téllez por su revisión del manuscrito y por sus atinadas sugerencias. Asimismo deseamos expresar nuestro reconocimiento a J. L. Villaseñor por la traducción de la diagnosis al latín y por sus sugerencias. Los herbarios MEXU, MICH y RSA-POM amablemente facilitaron los ejemplares revisados para este trabajo.

\section{LITERATURA CITADA}

Dorado, O. 1987. Brongniartia sousae (Fabaceae: Faboideae), a new species from the Isthmus of Tehuantepec, Oaxaca, México. Aliso 11: 631-634.

Dorado, O. 1988. A. morphological revision of the podalyrioides group of Brongniartia (Fabaceae: Faboideae). Master of Science Thesis. Claremont Graduate School. Claremont, California. 158 pp.

Dorado, O. 1989. Brongniartia vazquezii (Fabaceae: Faboideae), a new species from the state of Morelos, México. Syst. Bot. 14(1): 20-23.

Jiménez, J. y J. L. Contreras. 1989. Una especie nueva de Brongniartia (Leguminosae: Faboideae) de Guerrero, México. Anales Inst. Biol. Ser. Bot. UNAM (Núm. único) 58: 63-68.

McVaugh, R. 1987. Brongniartia, In: McVaugh, R. (ed). Flora Novo-Galiciana. University of Michigan Press. Ann Arbor. Vol. 5. pp. 297-321. 